Accretion Phenomena and Related Outflows, IAU Colloquium 163

ASP Conference Series, Vol. 121, 1997

D.T. Wickramasinghe, L. Ferrario, and G.V. Bicknell, eds.

\title{
Stability of Viscous Accretion Tori
}

\author{
R.Kleiber, W.Glatzel \\ Universitätssternwarte Göttingen \\ Geismarlandstr. 11 \\ 37083 Göttingen, Germany
}

\begin{abstract}
With respect to the stability of accretion tori the linear stability of infinitely long cylinders is investigated taking into account the effect of viscosity. Critical Reynolds numbers as a function of the Mach number and the azimuthal wavenumber of the perturbation are calculated.
\end{abstract}

\section{Introduction}

Since the discovery that accretion tori are unstable with respect to non-axisymmetric perturbations (Papaloizou \& Pringle, 1984) in all investigations of this instability the effect of viscosity has been ignored so far. Here we present the first results of an attempt to take viscosity into account in the stability analysis of accretion tori.

For the stability analysis the flow in accretion tori is approximated by a flow between infinitely long concentric cylinders, a restriction which in previous investigations was found to provide qualitatively correct results concerning the stability properties. In the present study we consider configurations having constant specific angular momentum distribution and constant shear and bulk viscosity. Moreover, self-gravity and the radial accretion flow are neglected. Energy equations and equation of state are condensed in a polytropic relation between pressure and density. The stationary configurations thus obtained (they are identical to those used by Glatzel, 1987) are characterized by the Mach number $M$ defined at the pressure maximum.

After linearisation the Navier-Stokes-Equations (together with the continuity equation) pose a sixth order boundary eigenvalue problem for the complex eigenfrequency. Parameters of the problem are the Mach number $M$, the Reynolds number $R e$ and the azimuthal wavenumber $m$. We emphasize that previous investigations correspond to an infinite Reynolds number.

\section{Results}

The main result of the stability analysis is condensed in Fig. 1 and shows the critical Reynolds number (neutral stability) as a function of $m$ for various Mach numbers. The curve of neutral stability divides the parameter space into a domain of stability (left) and instability (right).

Perturbations with $m>9$ are stable in thick configurations $(M<3)$ for 


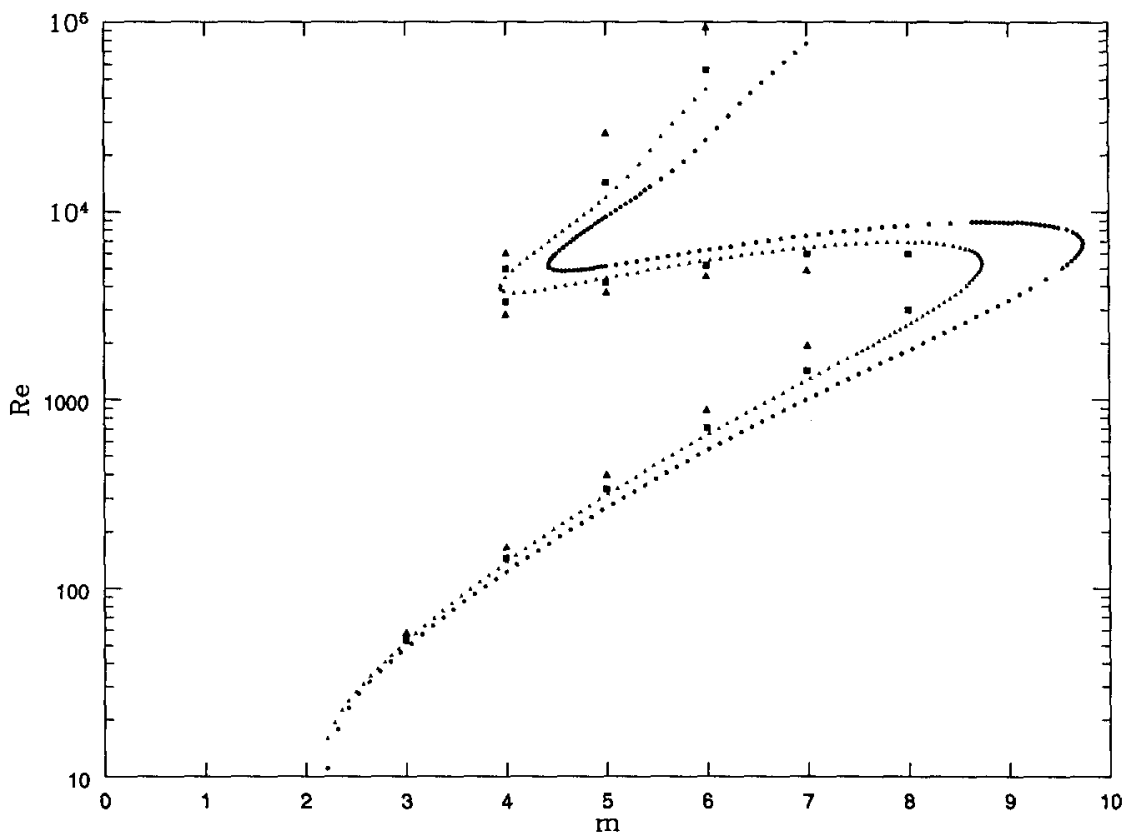

Figure 1. Curves of neutral stability for $M=3.0,2.5,2.3,1.9$ (right to left) corresponding to a ratio of the boundary radii of $7,20,70, \infty$.

Reynolds numbers less than $10^{5}$. The domain of stability is enhanced if rigid boundary conditions were used instead of free ones. In the range $2<m<10$ the critical Reynolds number lies between 50 and 7000 and depends strongly on the value of $m$. Perturbations with $m=1$ and $m=2$ remain unstable even for $R e=10$ (free boundary conditions). In the case of rigid boundary conditions they become stable at $R e \approx 10$ and 30 , respectively. The S-shape of the critical curves which may lead to three points of neutral stability for given $m$ is caused by the shear viscosity. This behaviour disappears if only bulk viscosity is considered.

\section{References}

Glatzel, W., 1987, MNRAS, 225, 227.

Papaloizou, J.C.B., Pringle J.E., 1984, MNRAS, 208, 721. 